\title{
Hybrid Architectures and Their Impact on Intelligent Design
}

\author{
Abe Kandel \\ Computer Science and Engineering Department \\ University of South Florida \\ Tampa, FL
}




\section{FUZZY HYBRID SYSTEMS \\ Critical Issues}

In this presentation we investigate a novel framework for the design of autonomous fuzzy intelligent systems. The system integrates the following modules into a single autonomous entity:

1) a fuzzy expert system

2) artificial neural network

3) genetic algorithm

4) case-base reasoning.

We describe the integration of these units into one intelligent structure and discuss potential applications.

- What are the benefits of fuzzy sets theory and fuzzy logic?

- Current and emerging technologies in fuzzy logic affect science and technology.

- What must be considered when designing systems in uncertain environments?

- How do we integrate fuzzy logic with intelligent systems, expert systems and neural networks? 


\section{FUZZY HYBRID SYSTEMS}

Here we address some of the issues involved in developing a technology that supports the implementation of an autonomous fuzzy intelligent system. The proposed technology is based on the premise that integrated solution architectures will be much more effective and highly flexible in their ability to successfully handle a broad base of applications with a wider scope of problem variations.

Hybrid systems [1] in artificial intelligence represent a new field of research that deals with the synergism of expert systems and neural network technologies. The integration of the computational paradigms of these two highly complementary knowledge representation techniques is imperative to the process of developing effective robust intelligent systems for a large number of important applications.

This idea of hybridization is carried one step further by also incorporating genetic algorithms and case-base reasoning into the autonomous fuzzy intelligent system.

In the following we briefly describe the nature of the modules and the principles involved in their integration. We conclude by indicating a high SIQ (System Intelligent Quotient) in the performance of the system as applied to image analysis.

- Fuzzy hybrid systems deal with the design of intelligent fuzzy integrated fuzzy systems (IHS) for real-time applications.

- Intelligent hybrid systems represent a new field of artificial intelligence research that is concerned with the synergism of expert systems, neural networks, genetic algorithms, case-base reasoning and fuzzy logic technologies. 


\section{INTEGRATED IMAGE PROCESSING ENVIRONMENT}

The autonomous fuzzy intelligent system (AFIS) architecture integrates the computational paradigms of fuzzy expert systems, neural network(s), genetic algorithm(s), and case-base reasoning. Expert systems and neural networks represent complementary approaches to knowledge representation; the logical, cognitive, and mechanical nature of the expert system versus the numeric, associative and self-organizing nature of the neural network.

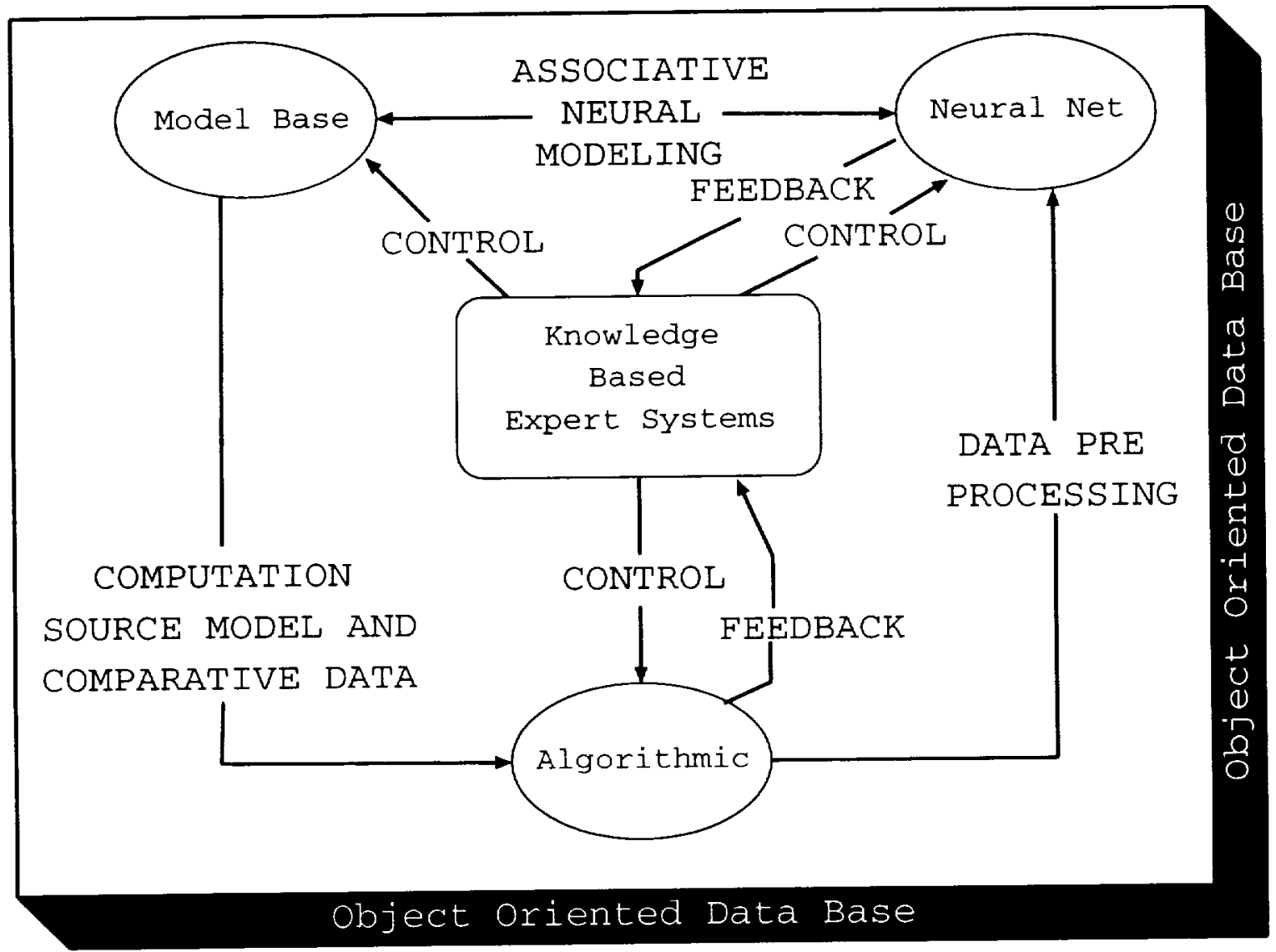




\section{NEURAL NETWORK AND EXPERT SYSTEM COMPARISONS}

Neural networks consist of densely interconnected simple nonlinear devices called processing elements (or neurons). The knowledge of a neural network resides in the interconnections between these elements and in the strengths (weights) of the interconnections. Collectively, neurons with simple properties, interacting according to simple rules, can accomplish complex functions such as generalization, error correction, information reconstruction, pattern analysis, and learning [2]. Neural networks provide a greater degree of robustness, or fault tolerance, than conventional von Neumann sequential machines. Indeed, damage to a few neurons or interconnections, and/or minor variabilities in the characteristics of neurons, do not impair overall network performance significantly. In addition, neural networks also possess the ability to gracefully handle inconsistencies or conflicts in the data.

Expert systems and artificial neural networks have unique and sometimes complementary features:

\section{Expert Systems}

Symbolic

Logical

Mechanical

Sequential

Closed

\section{Neural Networks}

Numeric

Associative

Biological

Parallel

Self-Organizing 


\section{MODELS FOR INTEGRATING EXPERT SYSTEMS AND NEURAL NETWORKS}

It is of paramount importance to develop methodologies for integrating the computational paradigms of fuzzy expert systems (FES), neural networks (NN), genetic algorithms (GA), and casebase reasoning (CBR) tools and for exploring the similarities between the underlying structure of these tools of knowledge representation. Such architecture, which allows for imprecise information and/or uncertain environments, is more powerful than either of its components standing alone.

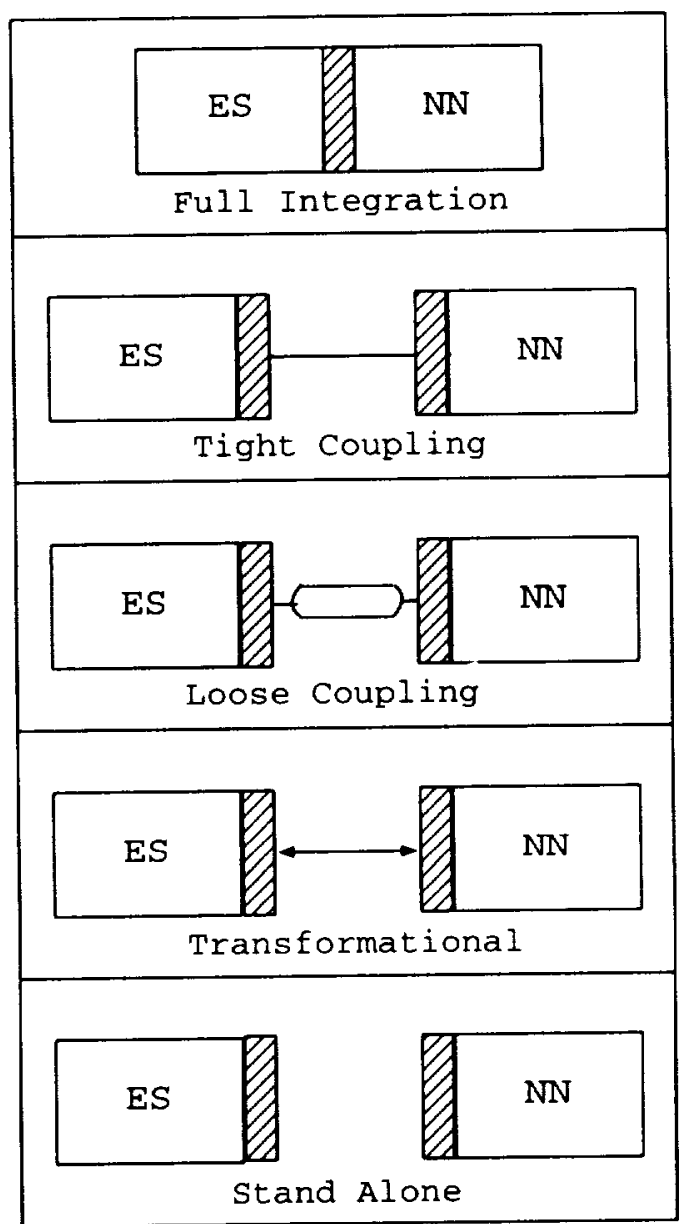




\section{HYBRID SYSTEMS}

The integrated system allows for a bidirectional (transparent) transfer of knowledge between its components. Initially, the fuzzy expert system is invoked with a base of knowledge which is necessarily incomplete. The neural network as well as the genetic algorithm and the CBR tool, take the knowledge from the fuzzy expert system and modifies it through learning. (Since the fuzzy expert system supplies the metaknowledge to begin the learning process, learning in the neural network is implemented more efficiently.) Based on the information acquired from the learning agents, the fuzzy expert system can modify certainty factors, modify existing rules, or infer new rules.

Thus, the ability of the system to learn in an imprecise environment enables the fuzzy expert system to modify and enrich its knowledge structures autonomously. Consequently, among other things, the AFIS can overcome some of the major drawbacks of conventional intelligent systems:

1) their reliance upon consultation with human experts for knowledge acquisition (the knowledge acquisition bottleneck);

2) their inability to synthesize new knowledge; and

3) their inability to allow for dynamic environments by changing knowledge whenever this becomes necessary.

- Computational intelligence

- Reinforcement learning

- Interactive AI

- Ability to generalize

- New insights

- Predictions

- Justification 


\section{FUZZY HYBRID SYSTEMS}

The system is functionally decomposed into four separate units which communicate by means of common data structures. The rule base is the common data structure between the fuzzy expert system and the neural network, whereas the collection of state arrays is the common data structure between the neural network and the learning unit.

- A variety of applications involve human expertise and knowledge which is imprecise, incomplete, or not totally reliable.

- To better emulate human decision-making processes and to allow for imprecise information and/or uncertain environments, the $I H S$ must combine knowledge-based techniques for gathering the processing information with methods of approximate reasoning.

- Fuzzy logic is of particular importance because it provides a systematic framework for dealing with fuzzy quantifiers. It thus subsumes both predicate logic and probability theory and makes it possible to deal with different types of uncertainty within a single conceptual framework. 


\section{HYBRID SYSTEMS}

The neural network component of the system consists of relatively small networks, each limited to performing very specific cognitive and recognition tasks. In this way, highly optimized network functions are achieved within manageable training and computational constraints. A variety of neural network paradigms are used according to the capability of each paradigm to optimally contribute to some facet of the exploitation problem. In particular, associative neural networks are used to provide high performance model storage mechanisms for model-driven recognition and classification.

The system provides a bidirectional transfer of knowledge between its components. Initially, the fuzzy expert system is invoked with a base of knowledge which is necessarily incomplete. The neural network takes the knowledge from the fuzzy expert system and modifies it through learning. Based on the information acquired from the neural network, the genetic algorithm and the CBR tool, the fuzzy expert system can modify certainty factors, modify existing rules, or infer new rules.

Thus, the learning capability of the system, provided by the neural network and the genetic algorithm, as well as with examples derived from the CBR, is used to acquire new knowledge from the environment, whereas the fuzzy expert system makes use of knowledge it had already acquired.

Therefore, the fuzzy expert system relieves the other component from learning things already known.

- Harnessing the combined power of fuzzy expert systems, neural networks, genetic algorithms and CBR in a hybrid system, which allows for imprecise information and/or uncertain environments, yields a system more powerful than either one of its components standing alone.

- Furthermore, such hybrid systems can undoubtedly offer improved solutions to the process of developing effective robust intelligent systems for a large number of important real-time applications. 


\section{LEARNING}

The integrated system merges knowledge-based, neural-based, algorithmic-based, and modelbased paradigms, all used to exploit the data. These paradigms are intimately bound and distributed throughout the general process of data exploitation. The elements of the system are integrated within an object-oriented process and data base environment.

Processes within the integrated environment are freely selected and concatenated based on the output data produced by the processes themselves. The exploitation process is guided by the knowledge base which uses the feedback generated by individual process execution to drive the choice and strategy of the next process initiated by the exploitation process.

Each of the processes acts in concert with the others. For example, algorithmic preprocessing of a given type may be initiated prior to the specific selection of an appropriate neural network architecture and trained weight set. A neural network process may feedback information to the knowledge-based system that would trigger an exploitation initiative using another more specialized neural network architecture or training set. Specific models and the manner in which they interplay with both neural network and algorithmic processes are guided by the knowledge-based system.

- Learning in uncertain or unknown environments, particularly autonomous or unsupervised learning, is an essential component of any intelligent system.

- The ability to garner new information, process it, and increase the understanding and capability of the system is crucial to the performance of autonomous intelligent systems.

- Such systems should be able to generalize and apply appropriate previous solution or hypothesize new solutions to situations never before encountered by the system. 


\begin{abstract}
APPLICATIONS
The key to the integrated image processing environment is that the knowledge-based system most predominantly drives the strategy of exploitation within the facilitating object-oriented environment. The object-oriented approach effectively implements a flexible and adaptable intelligent system that allows the flow and sharing of information and coordination of processes to enhance significantly the overall objective of the image.
\end{abstract}

1. Intelligent Robot Control Systems

An important issue related to robotic systems is that of spatial reasoning to support mobility in unpredictable and cluttered environments.

2. Command and Control Systems

The ideal command and control (C2) system should be able to respond quickly and accurately to situations requiring both complex reasoning processes and intensive computational analysis of data received from a variety of sensors.

3. Automatic/Intelligent Identification and Recognition 


\section{REFERENCES}

1. Kandel, A. and Langholz, Architectures for Hybrid Intelligent Systems, CRC Press, 1992.

2. Simpson, P. K., Artificial Neural Systems, Pergamon Press, New York, 1990. 